\title{
Renal Allograft Compartment Syndrome: A Case Report and Review of the Literature
}

\author{
Nasrin El-Bandar ${ }^{a}$ Markus Lerchbaumer ${ }^{b}$ Mandy Hubatsch ${ }^{a}$ \\ Isabel Michaela Lichy ${ }^{a}$ Matthias R.G. Schulz ${ }^{\text {a }}$ Frank Friedersdorff ${ }^{a}$ \\ aDepartment of Urology, Charité - Universitätsmedizin Berlin, Corporate Member of Freie Universität Berlin, \\ Humboldt-Universität zu Berlin and Berlin Institute of Health, Berlin, Germany; ${ }^{b}$ Department of Radiology, \\ Charité - Universitätsmedizin Berlin, Corporate Member of Freie Universität Berlin, Humboldt-Universität \\ zu Berlin and Berlin Institute of Health, Berlin, Germany
}

\section{Keywords}

Compartment syndrome $\cdot$ Kidney transplantation .

Transplant vein compression · Mesh

\section{Abstract}

Kidney transplantation is the treatment of choice for patients with ESRD. One rare complication after transplantation is the development of renal allograft compartment syndrome (RACS). We present a case of 1 patient who developed RACS due to compression of the transplant vein, which was then treated by salvaging the kidney transplant using urgent decompression surgery with mesh fascial closure. We postulate that this technique is safe and should be the treatment of choice for patients with RACS.

(c) 2020 S. Karger AG, Basel

\section{Introduction}

Renal transplantation remains the best treatment option for patients with ESRD [1]. After transplantation, the primary goal is to preserve graft function and to pre- vent occurrence of allograft dysfunction or even graft loss. Different postoperative complications related to the surgical procedure may compromise graft function - especially vascular and ureteral complications [2]. One scarcely reported complication in the immediate postoperative course is the development of renal allograft compartment syndrome (RACS) [3]. The exact pathophysiology of this phenomenon is unknown. However, there is a consensus that limited space in the retroperitoneal compartment may contribute to the development of RACS due to compression of the renal parenchyma or vessels - both resulting in a reduced perfusion of the renal graft and subsequent deterioration of graft function [4-7]. Immediate decompression is necessary whenever RACS is suspected. Various decompression techniques are described, including mesh closure, intraperitoneal placement of the graft, and permanent fasciotomy [2, 3, 5-7].

Current knowledge still lacks information about pathogenesis, diagnosis, and treatment of RACS. Therefore, we want to share our experience and describe the course of a patient who developed RACS due to compression of the transplant vein after kidney transplantation. karger@karger.com www.karger.com/uin

(C) 2020 S. Karger AG, Base

Karger!
Frank Friedersdorff

Department of Urology, Charité Universitätsmedizin Berlin Charitéplatz 1

DE-10117 Berlin (Germany)

Frank.Friedersdorff@ charite.de 

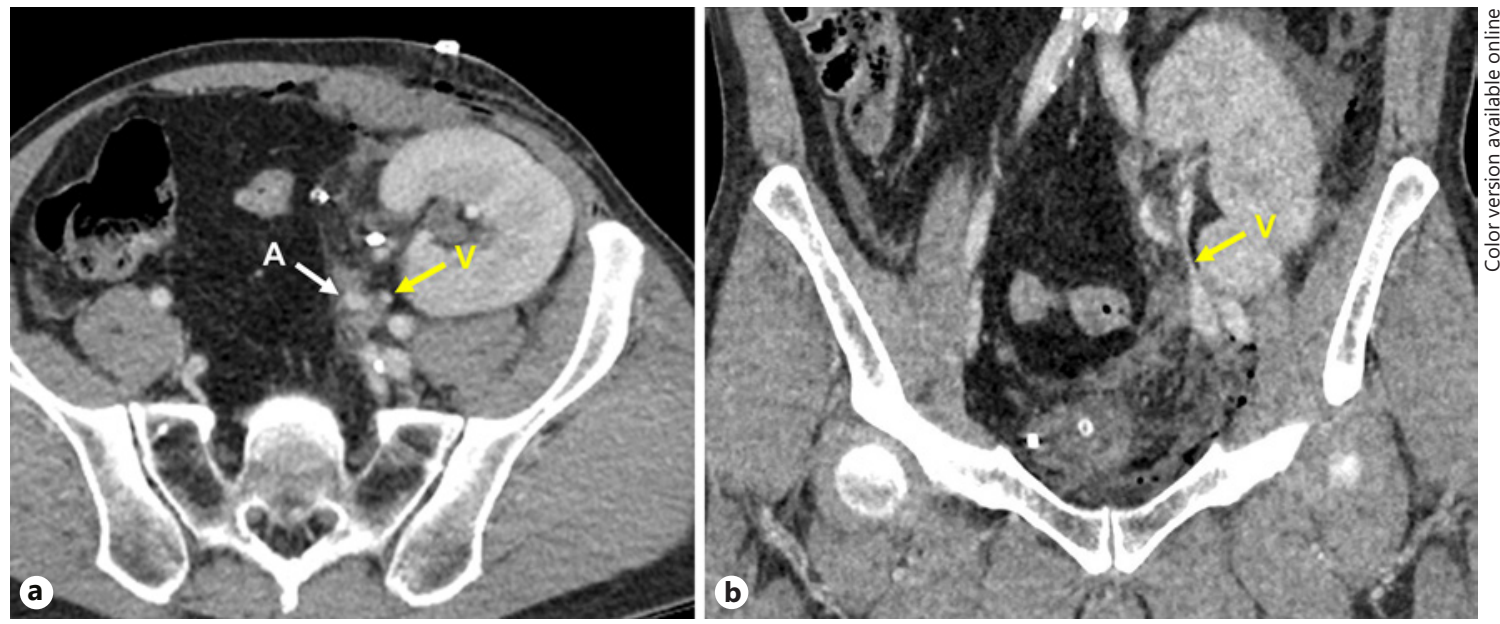

Fig. 1. Preoperative contrast-enhanced computed tomography (ceCT) scan with suspicion of venous outflow obstruction. Axial (a) and coronal (b) ceCT scan depicting the short-distance compressed narrow vein (V) with a lumen of $1 \mathrm{~mm}$ next to the transplant artery $(\mathrm{A})$.

Fig. 2. Postoperative contrast-enhanced computed tomography (ceCT) after revision surgery. Axial (a) and reconstructed sagittal (b) ceCT scan showing regular postoperative results of the repositioned transplant vein $(\mathrm{V})$ without compression after mesh placement.
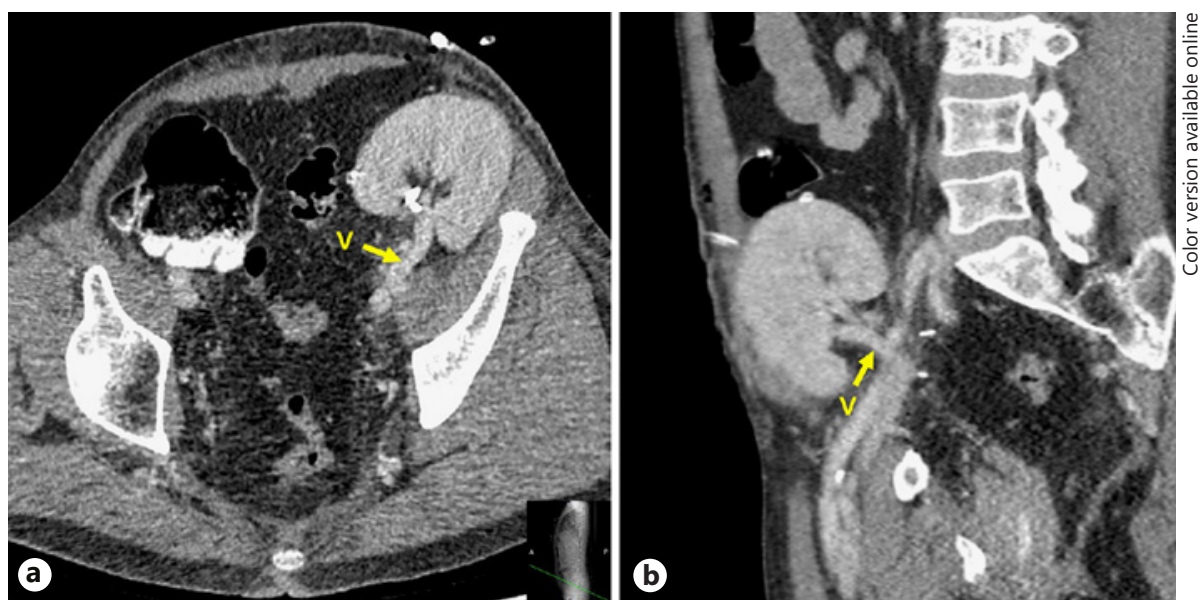

\section{Case Report}

We report the case of a 49 -year-old male patient receiving a renal graft from a deceased donor. The patient suffered from membranoproliferative glomerulonephritis as an underlying disease, eventually resulting into ESRD with subsequent need for dialysis and transplantation.

After 15 years of dialysis, kidney transplantation was performed successfully into the left iliac fossa with standard anastomosis technique, as previously described [8]. The kidney transplant was rather large with a pole-to-pole distance of $12 \mathrm{~cm}$, while the patient's body habitus was rather slim (height: $173 \mathrm{~cm}$; weight: $69 \mathrm{~kg}$ ). Surgery was performed without major complications or unusual findings. Only a small hematoma was registered at the inferior graft pole. However, during the immediate postoperative course, the patient remained anuric and dialysis was necessary. Routinely performed color-coded duplex sonography (CCDS) of the renal transplant showed reversed end-diastolic flow with high resistance index $(\mathrm{RI}>1)$, consistent with venous outflow obstruc- tion. Since CCDS of the renal transplant remained unchanged $4 \mathrm{~h}$ later, contrast-enhanced computed tomography (ceCT) was conducted. CeCT revealed compression of the narrow transplant vein $2 \mathrm{~cm}$ distal of the transplant hilum, showing a filiform transplant vein with only $1 \mathrm{~mm}$ diameter (Fig. 1). It also showed impaired cortical perfusion as a result of transplant vein compression and related venous congestion. Moreover, ceCT showed a $17-\mathrm{mm}$ hematoma at the inferior graft pole.

After interdisciplinary discussion, the patient was admitted to the operating theater for emergent revision surgery. Interestingly, after opening the wound, CCDS showed improved (i.e., normalized) blood flow. We assumed there was a mismatch between graft size on the one hand and retroperitoneal space on the other hand, resulting in compression of the transplant vein. In order to relieve the transplant vein from compression, the graft was placed in several different positions with subsequent partial fascial closure. However, immediately after partial fascial closure, CCDS repeatedly showed hampered blood flow. Thus, the operator decided to use a Vicryl mesh, which was sutured to the fascial edges for ten- 

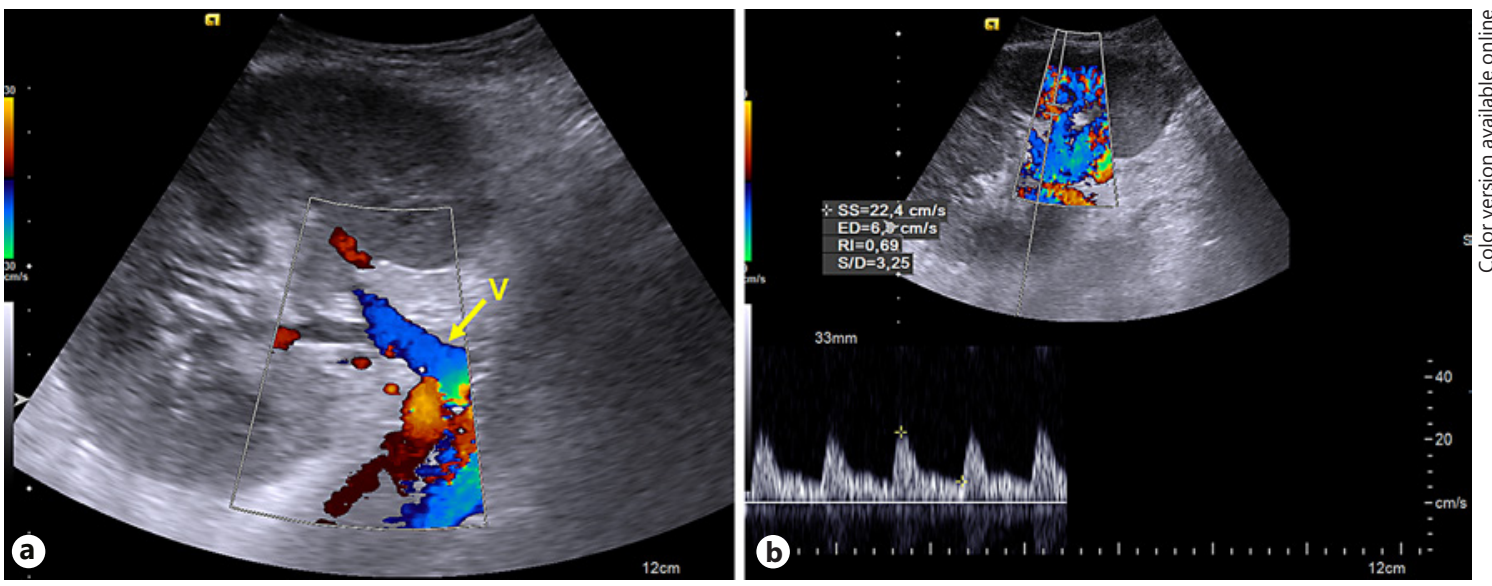

Fig. 3. Color-coded duplex sonography follow-up. Ultrasound follow-up performed 6 days after surgical repair showing good transplant vein outflow and optimized kidney transplant perfusion with intraparenchymal resistance indices of $0.69-0.75$.

sion-free closure of the fascial defect. After mesh placement, intraoperative CCDS presented a clearly improved flow profile. Postoperative ceCT scan revealed good renal outflow without depicting a stenosis of the repositioned transplant vein (Fig. 2).

During the following days after revision, blood flow remained constantly good in CCDS with the normal Doppler waveform pattern (RI < 0.8, Fig. 3). Nevertheless, due to delayed graft function, dialysis had to be conducted 4 times after transplantation.

Two days after revision surgery, the patient developed hospitalacquired pneumonia but recovered soon after antibiotic treatment. In the following days, both patient's condition and graft function improved. Serum creatinine decreased to $1.05 \mathrm{mg} / \mathrm{dL}$, and the patient was discharged from hospital 2 weeks after initial surgery for kidney transplantation. No further conspicuous findings and no deterioration of graft function occurred within 7 months follow-up.

\section{Discussion}

Among the current literature, occlusion of the transplant vein was often suspected to cause RACS $[5,9]$. However, to our knowledge, there is no case showing direct compression of the transplant vein in ceCT as the underlying cause for RACS. Patients with RACS were previously reported to show absent venous flow as a nonspecific sign in kidney ultrasound without direct evidence of venous compression $[9,10]$. We are the first to report direct evidence of venous compression in a transplant kidney with subsequent RACS.

Development of RACS is reported to be rare with an incidence of $2 \%$ [5]. However, the true incidence is suspected to be higher due to misdiagnosis. Graft loss or dys- function may often falsely be attributed to thrombosis or delayed graft function instead of RACS $[5,7]$.

Several aspects may contribute to misdiagnosis. On the one hand, CCDS in patients with RACS shows nonspecific findings as decreased/absent/reversed diastolic flow and high RI - these findings are also seen in acute tubular necrosis and transplant vein thrombosis [5-7, 9, $11]$. On the other hand, in cases of RACS, blood stasis occurs secondarily due to compression or occlusion of the transplant vein and may result in thrombus formation and transplant vein thrombosis [10]. Moreover, since this complication is thought to be rare, clinicians are not aware of it, which may result into delayed diagnosis or misdiagnosis that can lead to graft loss.

Pathogenesis of RACS is not clearly understood. RACS is related to the limited extraperitoneal space that is used as an artificially created graft compartment. Limited space may result in either parenchymal or vascular compression with subsequent decrease in graft perfusion and kidney function $[3,5,7]$. Especially, the transplant vein is at risk for compression - stenosis of the renal artery is rather reported to result from renal artery kinking [12]. If we define RACS as a subtype of abdominal compartment syndrome, we can assume pathogenesis is similar [6]. In 2 animal studies, Doty et al. $[13,14]$ investigated the role of renal parenchymal pressure and renal venous compression in the development of acute renal failure in abdominal compartment syndrome. They found out that external compression of the kidney plays a minor role compared to venous compression. Similarly, we can assume that compression of the transplant vein probably 
plays a more important role in the pathogenesis of RACS rather than parenchymal compression - as we have also seen in this case of our patient.

Immediate decompression is needed whenever RACS is suspected. Compression of the transplant vein can even be prevented by identification of risk factors for RACS during transplantation. Mismatch between a large kidney graft and a tight extraperitoneal compartment can lead to ischemia immediately after fascial closure $[6,7]$. Whenever ischemia is suspected and more force is needed for fascial closure, RACS may result. In these cases, suspicion can be confirmed by intraoperative CCDS [7]. Especially in combination with postoperative swelling of the renal transplant in the first weeks after transplantation, there is a need for sufficient space for the graft [11].

RACS either develops during initial surgery or is identified in the immediate postoperative course $[5,7]$. In our case, RACS was suspected due to findings in CCDS and ceCT after renal transplantation. In order to preserve graft function and relieve the renal transplant from venous congestion, decompression was indispensable, created by bridging the fascial defect with a Vicryl mesh. In literature, a mesh closure, intraperitonealization of the graft, and permanent fasciotomy are described for treatment of RACS [3, 5, 7]. Mesh closure is the preferred procedure, although a secondary infection because of the placement of a foreign body is feared [5, 7]. However, patients who received either intraperitonealization or permanent fasciotomy for treatment of RACS underwent hernia repair with mesh at a later time point [5]. A placement of mesh therefore seems unavoidable. Nevertheless, more information is needed in regard to which type of mesh we should use. In contrast to our approach, most authors used non-absorbable meshes for closure of the fascial defect $[3,6,15]$. We are the first to report the usage of Vicryl as an absorbable mesh for fascial closure. Absorbable meshes are assumed to be associated with less infection risk [16]. They are thought to act as a basis for creation of new tissue $[17,18]$. Further research is needed in order to determine the efficacy of this treatment and the material that should preferably be used.

\section{Conclusion}

Although RACS is a rare complication, it is suspected that the true incidence is higher than supposed. Therefore, clinicians need to be aware of this serious complication. Early detection of RACS by CCDS should prompt

urgent decompression surgery in order to salvage the graft and preserve graft function. Mesh closure is safe and should be the treatment of choice for patients with RACS.

\section{Statement of Ethics}

The patient has given his written informed consent to publish the case (including publication of images).

\section{Disclosure Statement}

The authors have no conflicts of interest to declare.

\section{Funding Sources}

The authors did not receive any funding for this case report.

\section{Author Contributions}

N.E.-B.: The author contributed to this study by conducting literature search, collecting data, interpretation of the data, and preparing the manuscript.

M.L.: The author contributed to this study by collecting data, interpretation of the data, editing the manuscript, and critical revision of the draft.

M.H.: The author contributed to this study by interpretation of the data and critical revision of the draft.

I.M.L.: The author contributed to this study by interpretation of the data and critical revision of the draft.

M.R.G.S.: The author contributed to this study by interpretation of the data and critical revision of the draft.

F.F.: The author contributed to this study by designing the study, interpretation of the data, editing the manuscript, and critical revision of the draft.

References

Urol Int 2020;104:646-650 DOI: $10.1159 / 000508799$
1 Nieuwenhuijs-Moeke GJ, Pischke SE, Berger SP, Sanders JSF, Pol RA, Struys MMRF, et al. Ischemia and reperfusion injury in kidney transplantation: relevant mechanisms in injury and repair. J Clin Med. 2020;9(1):253.

2 Halawa A. Abdominal wall closure of renal transplant recipients: an undermined challenge. Int J Organ Transplant Med. 2010;1(2): 73-6.

3 Beasley KA, McAlister VC, Luke PP. Mesh hood fascial closure in renal allograft compartment syndrome. Transplant Proc. 2003; 35(7):2418-9.

4 Nguan CY, Beasley KA, McAlister VC, Luke PP. Treatment of renal transplant complications with a mesh hood fascial closure technique. Am J Surg. 2007;193(1):119-21. 
5 Ball CG, Kirkpatrick AW, Yilmaz S, Monroy M, Nicolaou S, Salazar A. Renal allograft compartment syndrome: an underappreciated postoperative complication. Am J Surg. 2006; 191(5):619-24.

6 Heer MK, Trevillian PR, Hardy DB, Hibberd AD. Salvaging kidneys with renal allograft compartment syndrome. Transpl Int. 2012; 25(4):e47-9.

7 Wood LN, Yang W, Annamalai A. Mesh hood fascial closure is a safe alternative to prevent renal allograft compartment syndrome during kidney transplantation. Transplant Proc. 2015;47(6):1845-9.

8 El-Bandar N, Kroy DC, Fuller TF, Kramer J, Liefeldt L, Budde K, et al. Development of graft-site candidiasis in 3 solid organ transplant recipients from the same donor. Am J Case Rep. 2017;18:777-81.
9 Humar A, Sharpe J, Hollomby D. Salvage of a renal allograft with renal vein occlusion secondary to extrinsic compression. Am J Kidney Dis. 1996;28(4):622-3.

10 Wiebe S, Kellenberger CJ, Khoury A, Miller SF. Early Doppler changes in a renal transplant patient secondary to abdominal compartment syndrome. Pediatr Radiol. 2004; 34(5):432-4.

11 Kolofousi C, Stefanidis K, Cokkinos DD, Karakitsos D, Antypa E, Piperopoulos P. Ultrasonographic features of kidney transplants and their complications: an imaging review. ISRN Radiol. 2013;2013:480862.

12 Miah M, Madaan S, Kessel DJ, Newstead CG, Guleria S. Transplant renal artery kinking: a rare cause of early graft dysfunction. Nephrol Dial Transplant. 2004;19(7):1930-1.

13 Doty JM, Saggi BH, Sugerman HJ, Blocher CR, Pin R, Fakhry I, et al. Effect of increased renal venous pressure on renal function. J Trauma. 1999;47(6):1000-3.
14 Doty JM, Saggi BH, Blocher CR, Fakhry I, Gehr T, Sica D, et al. Effects of increased renal parenchymal pressure on renal function. J Trauma. 2000;48(5):874-7.

15 Maione C, Gambino G, Di Bona A, Luna E, Turco D, Scio A, et al. PTFE mesh in renal allograft compartment syndrome. Transplant Proc. 2006;38(4):1049-50.

16 Brown CN, Finch JG. Which mesh for hernia repair? Ann R Coll Surg Engl. 2010;92(4): $272-8$.

17 Öberg S, Andresen K, Rosenberg J. Absorbable meshes in inguinal hernia surgery: a systematic review and meta-analysis. Surg Innov. 2017;24(3):289-98.

18 Huang Q, Li J, Lau WY. Techniques for abdominal wall closure after damage control laparotomy: from temporary abdominal closure to early/delayed fascial closure: a review. Gastroenterol Res Pract. 2016;2016:2073260. 